\title{
Chirurgische Mission in einer humanitären Katastrophensituation
}

\section{Rudolf Baudenbacher}

Facharzt für Chirurgie, Schwerpunkt Allgemeinchirurgie und Traumatologie

\section{Berbérati / Zentralafrikanische Republik, an einem Morgen Mitte März 2014}

Wir - eine italienische Anästhesieärztin, meine Frau, OP-Pflegefachfrau, und ich - drei internationale Mitarbeitende von Médecins sans Frontières / Ärzte ohne Grenzen (MSF) Schweiz, verlassen kurz nach 7.30 Uhr unsere von einer hohen Mauer umgebene, Tag und Nacht beaufsichtigte Basis. Ein einheimischer Chauffeur fährt uns in einem geländegängigen Fahrzeug ins 500-600 Meter entfernte Hôpital Régional Universitaire. Ordnungsgemäss haben wir kurz nach Abfahrt über Funk der Radiozentrale gemeldet, welche Personen in besagtem Auto an Bord sind.

Auf der Strasse zum Spital, einer Hauptstrasse in der drittgrössten Stadt des Landes, sind an diesem
Morgen, völlig unafrikanisch, nur wenige Menschen, die meisten zu Fuss, unterwegs.

Zwar ist vom herrschenden Krieg unmittelbar nichts wahrzunehmen, nichtsdestotrotz ist die Ausnahmesituation für die Bevölkerung sogar im Vorbeifahren sicht- und spürbar. Im Spital müssen wir dem Chauffeur unsere Ankunft durch Unterschrift bestätigen. Vor der Operationsabteilung erwarten uns einige Mitarbeiter des OP-Teams.

MSF Schweiz hatte im Januar 2014 das Nothilfe-Projekt mit primär einer pädiatrischen Equipe im Hôpital Régional Universitaire in Berbérati gestartet: In einem grossen öffentlichen Spital, in dem eine funktionstüchtige Infrastruktur aber weitestgehend fehlte. MSF arbeitete ohne Röntgengerät, ohne
Korrespondenz: Ärzte ohne Grenzen Sibylle Berger Kanzleistrasse 126 CH-8026 Zürich

sibylle.berger[at]geneva.msf.org

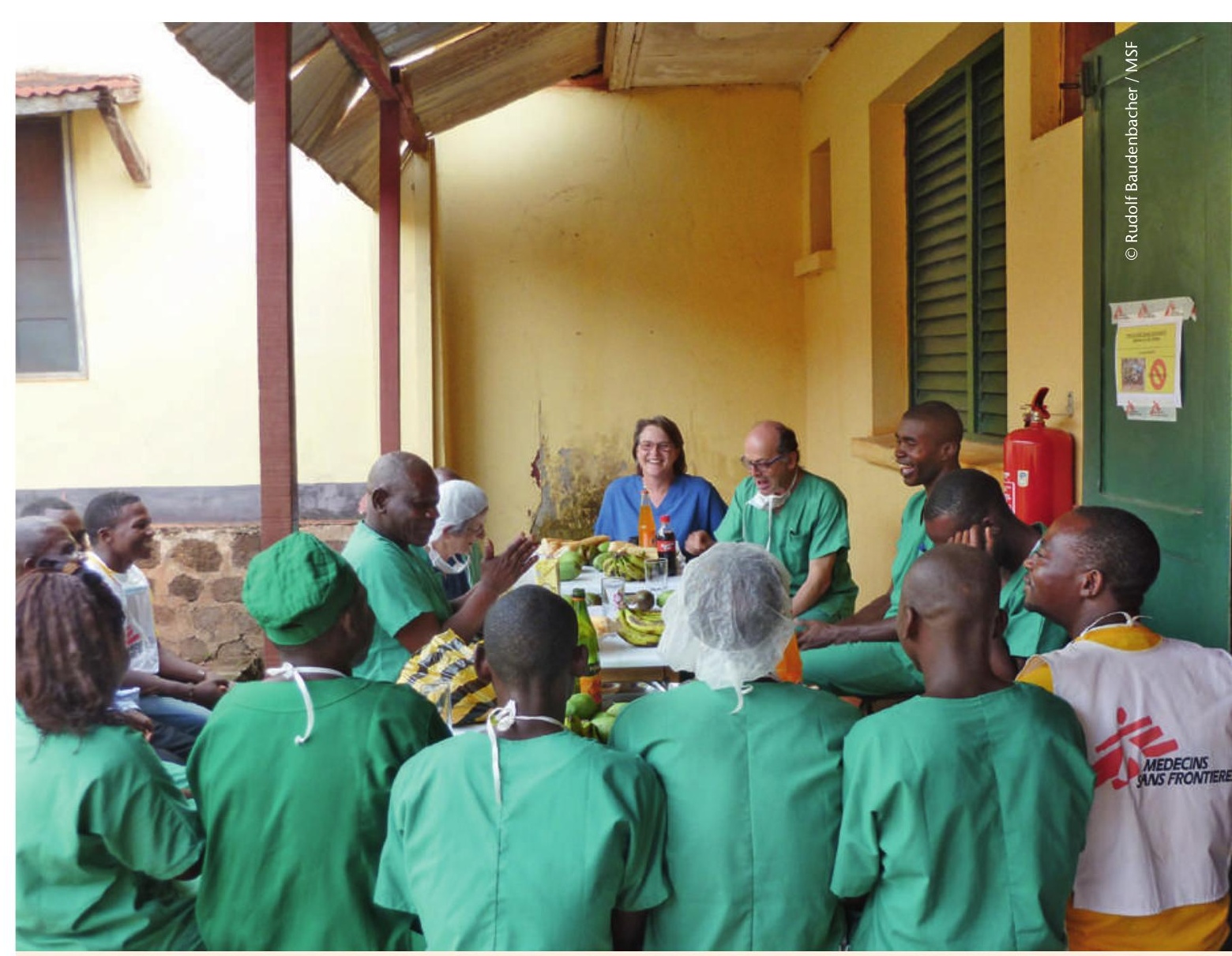

Das Team des «Hôpital Régional Universitaire Berbérati» in der Zentralafrikanischen Republik, wo seit Frühjahr 2013 Krieg herrscht. 


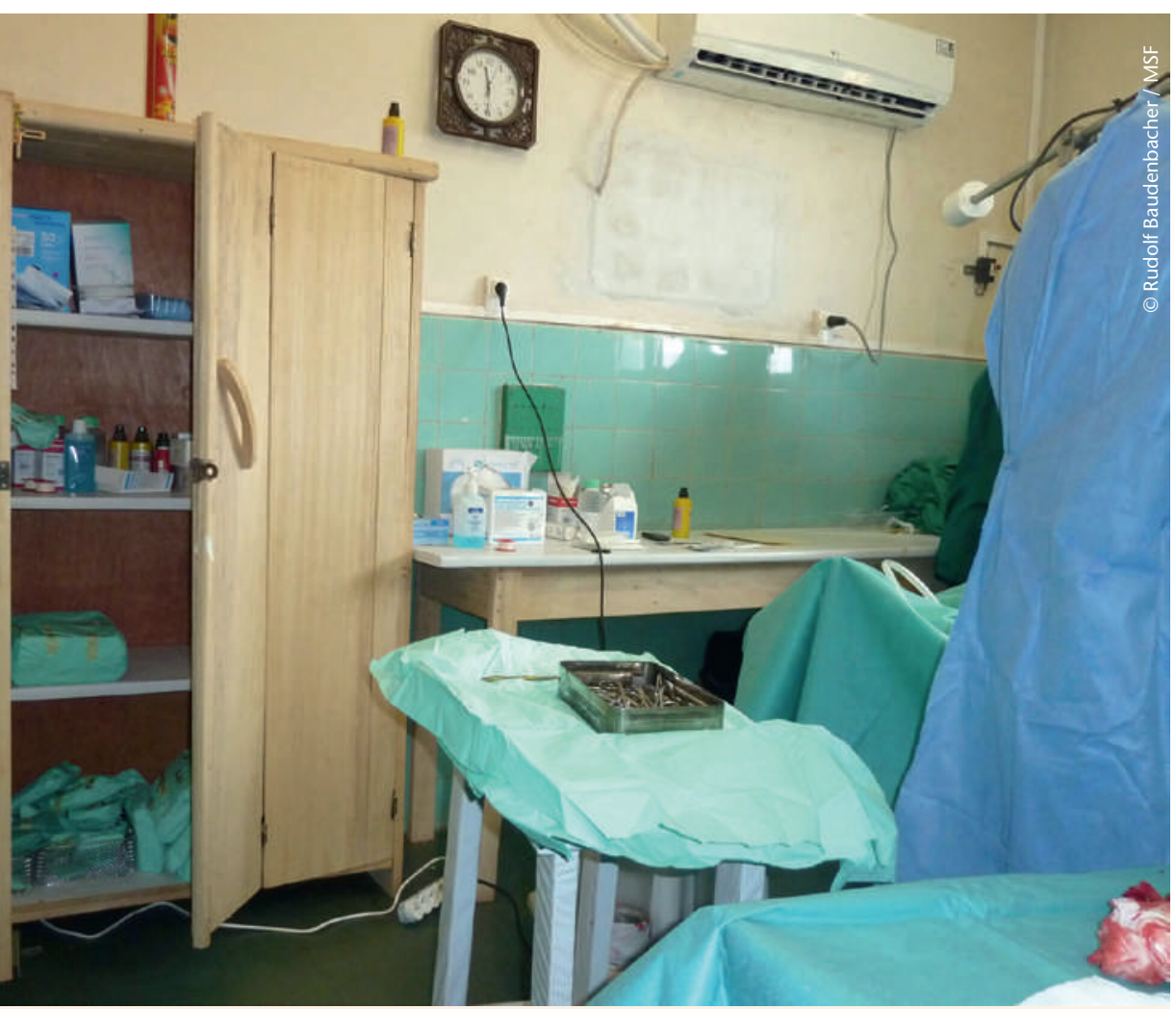

Die Ausstattung des Operationsraums wurde verbessert, doch vieles fehlt noch immer, z. B. gute Operationslampen und Klimaanlagen.

Labor, ohne geregelten Medikamenten-, Verband- und Verbrauchsmaterial-Nachschub, mit einer rudimentär ausgerüsteten Operationsabteilung ohne adäquate Sterilisationsmöglichkeit und mit Personal, dessen fachliche Qualifikation sich in vielen Fällen auf einem recht tiefen Niveau bewegte. Drei Wochen nach der pädiatrischen hatte die erste chirurgische Equipe - ein Chirurg, eine OP-Pflegefachfrau und eine Anästhesistin - ihre Arbeit in Angriff genommen.

Meine Frau, erfahren durch jahrelange Instrumentierund Operationspflegetätigkeit, nimmt mit dem einheimischen Personal, das heute im Dienst ist und aus lauter Männern besteht, die Arbeitsvorbereitungen in der Operationsabteilung auf. Letztere haben wir vor gut drei Wochen in einem hygienisch, infrastrukturell und funktionell noch erheblich verbesserungsnotwendigen Zustand vorgefunden. In der Zwischenzeit ist es dank vorzüglicher Zusammenarbeit mit den zuständigen Logistikern und deren Mitarbeitern gelungen, eine Sterilisationstätigkeit nach MSF-Richtlinien durch geschultes, einheimisches Personal aufzubauen, in den beiden Operationssälen eine hygienisch akzeptable und arbeitstechnisch sinnvolle Infrastruktur einzurichten, eine effiziente Reinigungsequipe zu rekrutieren und zu schulen sowie Arbeitsabläufe und Diensteinsatzzeiten des OP-Personals zu regeln.

Es fehlen nach wie vor Operationslampen, die den Namen verdienen, ausreichende Klimaanlagen, zusätzliche Instrumenten-Sets und vor allem die Mög- lichkeit einer systematischen Ausbildung des Personals auf allen Stufen.

Seit Frühling 2013 herrscht in der Zentralafrikanischen Republik Krieg. Die Konflikte zwischen muslimischen (Seleka) und christlichen (Anti-Balaka) Milizen haben die Staatsmacht lahmgelegt und Eingreiftruppen der französischen Armee (Sangaris), der afrikanischen Union (Misca) sowie die soweit noch vorhandene zentralafrikanische Armee (Faca) auf den Plan gerufen. In Berbérati, im Südwesten des Landes, haben wir den Krieg nur unterschwellig miterlebt. Einer unmittelbaren Bedrohung durch Bewaffnete sind wir nie ausgesetzt gewesen. In unregelmässigen Abständen sind jeweils Gruppen von Anti-Balaka-Milizen in der Region aufgetaucht. Einige Male ist es zu Schiessereien auf dem Spitalareal gekommen. Diese haben jeweils die unverzügliche Evakuation aller internationalen Mitarbeiter aus dem Spital zur Folge gehabt.

Zusammen mit der Anästhesistin mache ich zuerst die morgendliche Visite im Chirurgie-Pavillon, wo zwischen 30 und 40 Patientinnen und Patienten hospitalisiert sind. Es geht darum, für die nachts Aufgenommenen die therapeutischen Massnahmen festzulegen, sicherzustellen, dass die für heute programmierten Patienten bereit sind, und Verordnungen für den Tag vorzunehmen.

Wir beginnen unser Operationsprogramm. Ein Knabe mit bereits einmal behandelten Granatsplitterverletzungen an beiden Beinen wird vom einheimischen Anästhesiepfleger unter Anleitung durch die Anästhesieärztin narkotisiert. Die Verbände werden entfernt, die Wunden gereinigt, soweit nötig nachdebridiert und neue Verbände angelegt. Nach dem Erwachen kommt der Knabe in den Aufwachraum, von wo er später durch unsere Träger auf einer Bahre in die Chirurgie-Station zurückverlegt wird.

Unser zweiter Fall ist ein junger Mann mit Femurschaftschussfraktur, bei dem 10 Tage nach der Verletzung bei noch offenen, nicht infizierten Schusswunden die Fraktur mit einem Fixateur extern stabilisiert wird. Dabei stehen uns weder ein Röntgenbild vor noch eines nach der Fixation zur Verfügung! Kriegschirurgie unter sehr erschwerten Bedingungen.

Das Hôpital Régional Universitaire Berbérati hatte bis 2010 angeblich recht gut funktioniert. Üblicherweise müssen in vielen afrikanischen Spitälern Konsultationen, Medikamente, Pflege- und allfälliges Operationsmaterial zum Voraus bezahlt werden. Aus diesen Einnahmen finanziert das Spital die Infrastruktur und den Medikamenten- und Verbrauchsmaterialnachschub sowie die Löhne der Angestellten. Nach 2010 hatte offenbar in der Folge eines Direktionswechsels ein Teufelskreis mit Abnahme der Patientenzahlen, Verminderung der Einnahmen sowie Abwanderung von qualifiziertem Personal eingesetzt. Der Krieg hatte schliesslich diese Negativspirale zusätzlich beschleunigt, das öffentliche Gesundheitswesen weitgehend lahmgelegt und unter anderem dazu geführt, dass die Angestellten während Mona- 


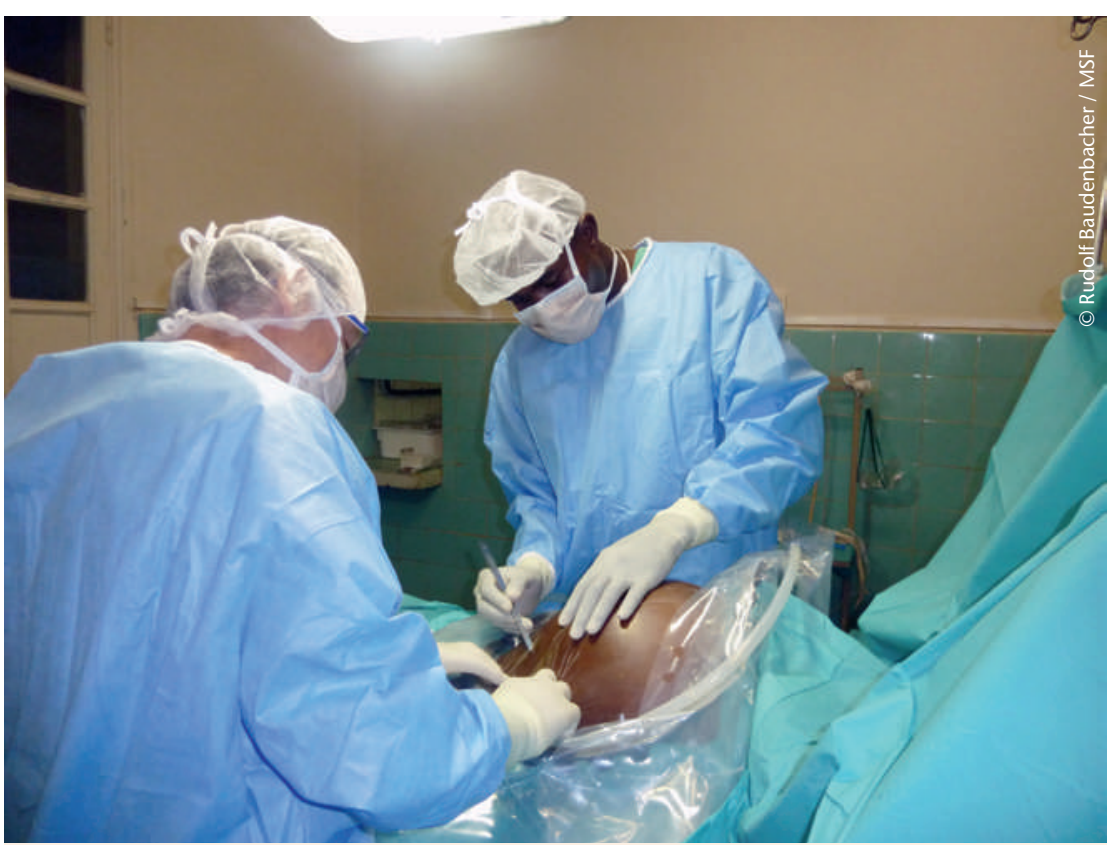

Immer wieder bringen Notfälle, hier ein Kaiserschnitt, das Operationsprogramm des Tages durcheinander.
Zwischenzeitlich wird gemeldet, dass in der Notfallstation ein Patient mit inkarzerierter Inguinalhernie zu beurteilen sei und von der pädiatrischen Poliklinik zwei Kleinkinder mit Abszessen und ein Kind mit Verbrennungen zur chirurgischen Behandlung geschickt worden seien. Nach einer Abszess-Sanierung und einer kurzen Mittagspause, die wir unserem Personal gewähren müssen, führen wir das Notfall-Programm, zu dem sich auch noch der Patient mit irreponibel inkarzerierter Inguinalhernie hinzufügt, bis in die Abendstunden weiter.

Den vier ursprünglich noch programmierten $\mathrm{Pa}$ tienten müssen wir klarmachen, dass sie heute nicht mehr operiert werden können. Sie werden für den kommenden Tag erneut ins Programm aufgenommen. Dafür, dass sie dann tatsächlich auch drankommen, besteht keine Gewähr! Eine für einen leidenden Patienten und auch für uns nahezu unerträgliche Situation.

Ein Einsatz in einer humanitären Katastrophensituation, wie wir sie im Spital von Berbérati angetroffen hatten, war und ist eine riesige Herausforderung. Unabdingbar sind eine langjährige und breite Berufserfahrung, Improvisationsfähigkeit, sichere Entscheidungskompetenz, Gelassenheit, Geduld, Selbstkritik und Respekt vor den zu behandelnden Menschen und den Mitarbeitenden, um nur einige Anforderungskriterien zu nennen.

Die grösste psychische Belastung stellte der nie endende Zustrom schwer erkrankter und verletzter Notfallpatientinnen und -patienten dar. Nicht wenige der Patienten konnten mangels zeitlicher oder materieller Ressourcen lediglich verzögert oder gar nicht behandelt werden. Kommt dazu, dass an eine Behandlung all der Leiden,

\section{«Den vier ursprünglich noch programmierten Patienten müssen wir klarmachen, dass sie heute nicht mehr operiert werden können.»}

Während der Vorbereitung für den dritten Eingriff des Tagesprogrammes wird von der Hebamme eine notfallmässige Sectio Caesarea angemeldet. Es wird sofort auf operative Geburtshilfe umgestellt. Der Kaiserschnitt entpuppt sich als sehr dringlich, ist doch der Kopf des Fötus nach einem bisher sehr langen Geburtsverlauf massiv im kleinen Becken der Mutter eingeklemmt; die Entwicklung des Kindes bereitet erhebliche Schwierigkeiten. die unbedingt einer elektiven Therapie oder Operation bedurft hätten, während unseres Einsatzes schlicht nicht zu denken war!

Warum müssen so viele Menschen in der Zentralafrikanischen Republik meist unverschuldet derart leiden? Auch für MSF ist es schwer, auf diese Frage eine umfassende Antwort zu finden. Aber die Mitarbeitenden von MSF in den Hauptquartieren sowie in den Einsatzländern vor Ort haben den Willen, diesen Menschen zu helfen. Zum Glück. 